\title{
Antibodies against immunogenic epitopes with high sequence identity to SARS-CoV-2 in patients with autoimmune dermatomyositis
}

Idiopathic inflammatory myopathies (IIMs) are rare, heterogeneous, autoimmune musculoskeletal diseases, characterised clinically by muscle weakness. Extramuscular involvement includes the skin, respiratory and cardiovascular systems. Genetic and environmental factors contribute to IIM susceptibility, and viral or bacterial infection may contribute to disease pathogenesis.

Both the innate and adaptive immune systems are important in IIM pathology. Two-thirds of affected individuals have known myositis-specific or associated autoantibodies, often linked to particular clinical features, ${ }^{1}$ and directed against proteins involved in key intracellular processes. Interferon pathways are differentially activated in clinical subtypes of myositis ${ }^{2}$; this interferon response is critical to protect the host against viral infection and modulate the antiviral immune response.

We recently used a high-throughput approach combining disease-specific immunoglobulin epitope signature enrichment and antigen identification from the total microbial 'exposome' (including viruses, bacteria, archaea and fungi) and human proteins. ${ }^{3}$ We applied this serum antibody repertoire analysis pipeline to investigate the microbial and autoantigen antibody repertoire accumulated throughout life in 20 adult-onset dermatomyositis patients seropositive for TIF $1 \gamma$ (TRIM33) autoantibodies, compared with 20 age-matched and gendermatched healthy controls. ${ }^{3}$ 
Table 1 Immunogenic epitopes enriched in dermatomyositis patients with sequence identity between bat coronavirus and SARS-CoV-2

\begin{tabular}{|c|c|c|c|c|c|c|}
\hline Bat coronavirus strain/epitope & Immunogenic epitope & $\begin{array}{l}\text { Human SARS-CoV-2 } \\
\text { sequence identity } \\
\text { (YP_009724389.1) }\end{array}$ & Length & Start-end (AA) & RefSeq protein & Protein \\
\hline BtCoV/279/2005_EP1 & VKGECPVMAPRR & GECP* & 4 & $268-271$ & YP_009725298.1 & Non-structural protein 2 \\
\hline \multirow[t]{2}{*}{ BtCoV/279/2005_EP2 } & SQAWQPLRQR & SQAWQP† & 6 & $6800-6805$ & YP_009725311.1 & 2'-0-ribose methyltransferase \\
\hline & & $\mathrm{LRQ}^{*}$ & 3 & $6883-6885$ & YP_009725311.1 & 2'-0-ribose methyltransferase \\
\hline BtCoV/279/2005_EP4 & DDAVVCVHGL & DDAVVC $†$ & 6 & $5152-5157$ & YP_009725307.1 & $\begin{array}{l}\text { RNA-dependent RNA } \\
\text { polymerase }\end{array}$ \\
\hline BtCoV/279/2005_EP5 & GGAVCRERPVS & GGAVCR† & 6 & $6405-6410$ & YP_009725309.1 & 3'-to-5' exonuclease \\
\hline Bat_SARS_CoV_Rm1/2004_EP1 & VKGECPVMAPRR & GECP* & 4 & $268-271$ & YP_009725298.1 & Non-structural protein 2 \\
\hline \multirow[t]{2}{*}{ Bat_SARS_CoV_Rm1/2004_EP2 } & SQAWQPLRQR & SQAWQP† & 6 & $6800-6805$ & YP_009725311.1 & 2'-0-ribose methyltransferase \\
\hline & & $\mathrm{LRQ}^{*}$ & 3 & $6883-6885$ & YP_009725311.1 & 2'-0-ribose methyltransferase \\
\hline Bat_SARS_CoV_Rm1/2004_EP3 & DDAVVCVHGL & DDAVVC $†$ & 6 & $5152-5157$ & YP_009725307.1 & $\begin{array}{l}\text { RNA-dependent RNA } \\
\text { polymerase }\end{array}$ \\
\hline Bat_SARS_CoV_Rm1/2004_EP4 & GGAVCRERPVS & GGAVCR $†$ & 6 & $6405-6410$ & YP_009725309.1 & 3'-to-5' exonuclease \\
\hline Bat_coronavirus_HKU5-5_EP2 & SAGCFVGLPIAG & AGCFV $¥$ & 4 & $5232-5236$ & YP_009725307.1 & $\begin{array}{l}\text { RNA-dependent RNA } \\
\text { polymerase }\end{array}$ \\
\hline Bat_coronavirus_HKU5-5_EP3 & WGAVCRKRPVS & GAVCR $\ddagger$ & 5 & $6406-6410$ & YP_009725309.1 & 3'-to-5' exonuclease \\
\hline
\end{tabular}

Disease-specific immunogenic epitopes identified against which immunoglobulins were raised. Cross-species alignment carried out using Clustal-0 and CLC Genomics Workbench 12.

*Blastp: not available

†Blastp: High specificity for SARS-CoV-2

$\ddagger$ Blastp: Low specificity for SARS-CoV-2

AA, amino acid; SARS-CoV-2, severe acute respiratory syndrome coronavirus-2.

Human coronaviruses are associated with the common cold, but can lead to fatal inflammatory responses and acute lung injury. Emergence of a novel coronavirus has caused a recent global pandemic of severe acute respiratory syndrome (SARS) in humans (COVID-19). ${ }^{4}$ Whole genome phylogenetic analyses suggest that the COVID-19 coronavirus SARS-CoV-2 shares high sequence similarity with bat coronaviruses and the host reservoir is bats. ${ }^{4}$

Due to the current coronavirus pandemic, here, we focused our analysis on epitopes mapping to the coronaviridae family. In dermatomyositis patients ${ }^{3}$, we identified enrichment of immunogenic linear epitopes (minimum 10 consecutive amino acids) mapping to 20 coronaviridae species, including 10 discrete epitopes mapping to three bat-coronavirus species. To investigate whether these 10 bat-coronavirus epitopes share sequence identity with human SARS-CoV-2, we carried out local alignment of the identified epitope sequences and the orf1ab polyprotein of SARS-CoV-2 (NCBI RefSeq: YP_009724389.1), and identified six distinct epitopes with high sequence identity (table 1). The epitopes were further queried against the database of non-redundant protein sequences (NCBI Blastp suite). Three linear epitopes of six amino acid length were highly specific for SARS-CoV-2 (table 1, figure 1). These epitopes map to SARS-CoV-2 2'-O-ribose methyltransferase, RNA-dependent RNA polymerase and 3'-to-5' exonuclease proteins. All three epitopes show extremely high conservation among currently available SARS-CoV-2 polyprotein sequences from the NCBI database (NCBI Multiple Alignment).

We subsequently investigated whether these epitopes have been experimentally identified as $\mathrm{B}$ cell and $\mathrm{T}$ cell immunogenic epitopes from studies of epidemic-causing virus SARS-CoV, or computationally predicted from SARS-CoV-2. Epitope 'DDAVVC' in the RNA-dependent RNA polymerase protein is a highly ranked CD8 $\mathrm{T}$ cell predicted epitope identified from the Immune Epitope Database and Analysis Resource, showing HLA-A*01:01 restriction. ${ }^{5}$

The coronavirus genome encodes four structural proteins; spike, nucleocapsid, membrane and envelope proteins. SARS-CoV-2 spike glycoproteins promote cell entry through attachment to the host ACE 2 receptor, and subsequent fusion between viral and host cell membranes to facilitate viral entry, ${ }^{6}$ and are the main target of antibodies. Here, we report identification of three immunogenic linear epitopes with high sequence identity to SARS-CoV-2 proteins in patients with autoimmune dermatomyositis, including 'DDAVVC' in the RNA-dependent RNA polymerase protein previously predicted as a CD8 T cell epitope, ${ }^{5}$ in keeping with $\mathrm{T}$ cell antigen presentation derived from processing both structural and non-structural proteins. The identification of immunogenic coronavirus epitopes with high sequence identity may indicate SARS-CoV-2 targets for vaccine development against COVID-19. Latent exposure to the coronaviridae family might contribute to musculoskeletal autoimmune disease development, as illustrated by a recent report of myositis in a patient with COVID-19.?

Spyridon Megremis, ${ }^{1}$ Thomas D J Walker, ${ }^{2}$ Xiaotong He, ${ }^{2}$

William E R Ollier, ${ }^{3,4}$ Hector Chinoy $\odot{ }^{5,6}$ Lynne Hampson, ${ }^{2}$ Ian Hampson, ${ }^{2}$ Janine A Lamb ${ }^{3}{ }^{3}$

'Division of Evolution and Genomic Sciences, The University of Manchester, Manchester, UK

${ }^{2}$ Division of Cancer Sciences, The University of Manchester, Manchester, UK ${ }^{3}$ Division of Population Health, Health Services Research and Primary Care, The University of Manchester, Manchester, UK

${ }^{4}$ Centre for Bioscience, Faculty of Science and Engineering, Manchester Metropolitan University, Manchester, UK

${ }^{5}$ National Institute for Health Research Manchester Biomedical Research Centre, Manchester University NHS Foundation Trust, The University of Manchester,

Manchester, UK

${ }^{6}$ Department of Rheumatology, Manchester Academic Health Science Centre, Salford Royal NHS Foundation Trust, Salford, UK 
B.

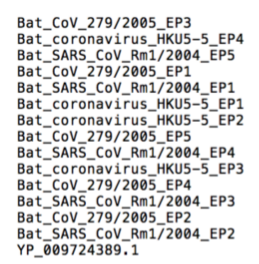

Bat_CoV_279/2005_EP3

Bat_coronavirus_HKUS-5_EP4
Bat_SARS_COV_Rm1 $1 / 2004$ EPP5

Bat_COV $-279 / 2005$ EP1

Bat_SARS_CoV_Rm1/2004_EP1
Bat_coronavirus_HKU5-5.EP1

Bat_coronavi rus_HKU5-5_EP
Bat_CoV_279/2005_EP5

Bat_SARS_COV_Rm1/2004_EP4

Bat_coronavirus_HKU5-5_EP3

Bat_SARS COV_Rm1/2004_EP

Bat_COV_279/2005_EP2
Bat_SARS_COV_Rm1/2004_EP2

Figure 1 Coronavirus species and epitopes. (A) Taxonomy tree of

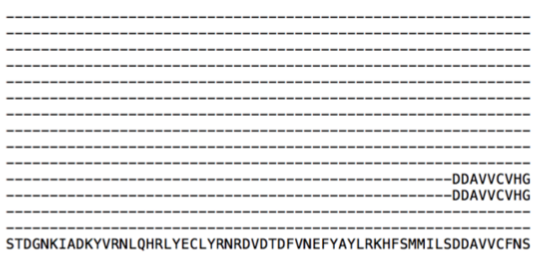

STDGNKIADKYVRNLOHRLYECLYRNRDVDTDFVNEFYAYLRKHFSMMILSDDAVVCFNS

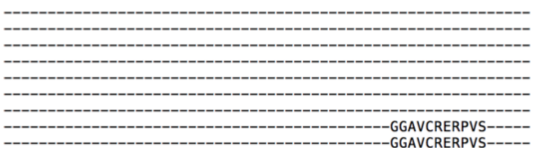

-GGAVCRKRPVS

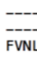

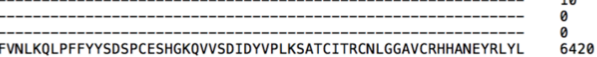
sequencing (NGS) reads per species is visualised as red bar plots. Green squares: presence only in dermatomyositis (DM), squares with no filling: presence only in healthy controls (HC). (B) Partial alignments of the three bat coronavirus epitopes which are shared with SARS-CoV-2. SARS-CoV-2, severe acute respiratory syndrome coronavirus-2.

Correspondence to Dr Janine A Lamb, Division of Population Health, Health Services Research and Primary Care, The University of Manchester, Manchester M13 9PL, UK; janine.lamb@manchester.ac.uk

\section{Handling editor Josef S Smolen}

Twitter Hector Chinoy @drhectorchinoy

Contributors Conceptualisation: IH, LH, TDJW, XH, SM, WERO and JAL. Methodology: IH, LH, TDJW, XH and SM. Software: TDJW and XH. Investigation: SM. Resources: IH, LH. Writing-original draft: SM and JAL. Writing-review and editing: SM, TDJW, JAL, IH, LH, XH, WERO and HC. Funding acquisition: JAL, IH, WERO and HC.

Funding This study was supported by a research grant from The Myositis Association. TDJW was supported by a research grant from Children with Cancer and The Caring Cancer Trust. XH was supported by grants from The Humane Research Trust and work in the Viral Oncology Labs was supported by grants from the Cancer Prevention Research Trust. HC and JL were supported by the Medical Research Council (MR/N003322/1). HC was supported by the NIHR Biomedical Research Centre Funding Scheme. The views expressed in this publication are those of the authors and not necessarily those of the NHS, the National Institute for Health Research or the Department of Health.

Competing interests JAL reports grants from The Myositis Association, grants from Medical Research Council (MR/N003322/1), during the conduct of the study; grants from Medlmmune, outside the submitted work.

Patient and public involvement Patients and/or the public were not involved in the design, or conduct, or reporting, or dissemination plans of this research.

Patient consent for publication Not required.

Ethics approval All samples were collected with relevant research ethics committee approval (MREC 98//8/86 North West Haydock Research Ethics Committee for UKMyoNet and UREC 02225 and UREC4 2017-1256-2489 for healthy control cohort).
Provenance and peer review Not commissioned; externally peer reviewed.

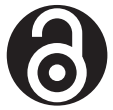

\section{OPEN ACCESS}

Open access This is an open access article distributed in accordance with the Creative Commons Attribution 4.0 Unported (CC BY 4.0) license, which permits others to copy, redistribute, remix, transform and build upon this work for any purpose, provided the original work is properly cited, a link to the licence is given, and indication of whether changes were made. See: https://creativecommons.org/ licenses/by/4.0/.

(C) Author(s) (or their employer(s)) 2020. Re-use permitted under CC BY. Published by BMJ.

\section{Check for updates}

To cite Megremis S, Walker TDJ, He X, et al. Ann Rheum Dis 2020;79:1383-1386.

Received 7 April 2020

Revised 4 May 2020

Accepted 5 May 2020

Published Online First 22 May 2020

Ann Rheum Dis 2020;79:1383-1386. doi:10.1136/annrheumdis-2020-217522

\section{ORCID iDs}

Hector Chinoy http://orcid.org/0000-0001-6492-1288

Janine A Lamb http://orcid.org/0000-0002-7248-0539 


\section{REFERENCES}

1 Betteridge Z, Tansley S, Shaddick G, et al. Frequency, mutual exclusivity and clinical associations of myositis autoantibodies in a combined European cohort of idiopathic inflammatory myopathy patients. J Autoimmun 2019;101:48-55.

2 Pinal-Fernandez I, Casal-Dominguez M, Derfoul A, et al. Identification of distinctive interferon gene signatures in different types of myositis. Neurology 2019;93:e1193-204.

3 Megremis S, Walker TDJ, He X, et al. Microbial and autoantibody immunogenic repertoires in TIF1 $\gamma$ autoantibody positive dermatomyositis. BioRXiv 2020.

4 Zhou P, Yang X-L, Wang X-G, et al. A pneumonia outbreak associated with a new coronavirus of probable bat origin. Nature 2020;579:270-3.

5 Grifoni A, Sidney J, Zhang Y, et al. A sequence homology and bioinformatic approach can predict candidate targets for immune responses to SARS-CoV-2. Cell Host Microbe 2020;27:671-80.

6 Letko M, Marzi A, Munster V. Functional assessment of cell entry and receptor usage for SARS-CoV-2 and other lineage B betacoronaviruses. Nat Microbio/ 2020;5:562-9.

7 Beydon M, Chevalier K, Al Tabaa 0, et al. Myositis as a manifestation of SARS-CoV-2. Ann Rheum Dis 2020. doi:10.1136/annrheumdis-2020-217573. [Epub ahead of print: 23 Apr 2020]. 